\title{
Impulsively Generated Linear and Non-Linear Alfvén Waves in the Coronal Funnels
}

\author{
P. Chmielewski ${ }^{a}$, A.K. Srivastava $^{b}$, K. Murawski $^{a}$ And Z.E. Musielak ${ }^{c, d}$ \\ ${ }^{a}$ Group of Astrophysics, UMCS, E. Radziszewskiego 10, 20-031 Lublin, Poland \\ ${ }^{b}$ Aryabhatta Research Institute of Observational Sciences (ARIES), Manora Peak, Nainital-263 129, India \\ and Department of Physics, Indian Institute of Technology (IIT)-BHU, Varanasi-221005, India \\ ${ }^{c}$ Department of Physics, University of Texas at Arlington, Arlington, TX 76019, USA \\ ${ }^{d}$ Kiepenheuer-Institut für Sonnenphysik, Schöneckstr. 6, 79104 Freiburg, Germany
}

(Received July 2, 2013; in final form December 4, 2013)

\begin{abstract}
We present simulation results of the impulsively generated linear and non-linear Alfvén waves in the weakly curved coronal magnetic flux-tubes (coronal funnels) and discuss their implications for the coronal heating and solar wind acceleration. We solve numerically the time-dependent magnetohydrodynamic equations to find the temporal signatures of the small and large-amplitude Alfvén waves in the model atmosphere of open and expanding magnetic field configuration with a realistic temperature distribution. We compute the maximum transversal velocity of both linear and non-linear Alfvén waves at different heights of the model atmosphere, and study their response in the solar corona during the time of their propagation. We infer that the pulse-driven non-linear Alfvén waves may carry sufficient wave energy fluxes to heat the coronal funnels and also to power the solar wind that originates in these funnels. Our study of linear Alfvén waves shows that they can contribute only to the plasma dynamics and heating of the funnel-like magnetic flux-tubes associated with the polar coronal holes.
\end{abstract}

DOI: $10.12693 /$ APhysPolA.125.158

PACS: 96.60.P-, 96.60.pc

\section{Introduction}

Small-amplitude (linear) Alfvén waves are incompressible magnetohydrodynamic (MHD) waves that propagate along the magnetic field lines, displacing them perpendicularly to the direction of wave propagation. The Alfvén waves carry energy from the solar sub-surface layers to its outer atmosphere [1]. Although, the theory of the linear Alfvén waves has been well established since the Nobel Prize discovery of Hannes Alfvén [2], the observations of these waves are difficult in the solar atmosphere due to their incompressible nature. There is indirect evidence for the presence of these waves in the solar atmosphere given by the SOHO and TRACE observations. However, more direct evidence for the existence of such transverse waves (e.g., Alfvén waves) in different magnetic structures of the solar atmosphere was supplied by high-resolution observations recorded by the Solar Optical Telescope (SOT) and the X-ray Telescope (XRT) on board of the Hinode Solar Observatory. According to the discovery made by Okamoto et al. [3], De Pontieu et al. [4] and Cirtain et al. [5], the signature of the Alfvén waves were observed in prominences, spicules, and X-ray jets, respectively, in the solar atmosphere using these instruments. Interpretations of these observations in terms of Alfvén waves is still not commonly accepted, see for example Erdélyi and Fedun [6], Van Doorsselaere et al. [7] and Goossens et al. [8, 9] who concluded that the observed waves were rather magnetoacoustic kink waves instead of being pure incompressible Alfvén waves. However, more recent arguments by Goossens et al. [9] seem to clearly indicate that the original interpretation of the observations in terms of incompressible Alfvén waves was indeed correct. Additional observational support was given by Tian et al. [10] who spectroscopically detected Alfvén waves.

Observational signature for the existence of torsional Alfvén waves in the confined magnetic fluxtube of the lower solar atmosphere was also reported by Jess et al. [11], who analyzed $\mathrm{H}_{\alpha}$ observations recorded with high spatial resolution by the Swedish Solar Telescope (SST). They interpreted and described the unique observations SST in terms of Alfvén waves in the localized chromosphere with periods from 12 min down to the sampling limit of the recorded observations near 2 min, with maximum power near 6-7 min. Tomczyk et al. [12] also reported on the ubiquitous presence of the Alfvén waves in the large-scale corona using the ground based observations of Coronal Multi-channel Polarimeter.

Extensive theoretical and observational studies of Alfvén waves are important in the context of te Sun because these waves are likely candidates for the atmospheric heating as well as supersonic wind acceleration in its polar coronal holes [13-15]. In such open field regions on the polar cap of the Sun, the non-thermal broadening of spectral lines was observed [16-21], which indicates the process of exchanging energy from Alfvén waves to the ambient plasma does occur in these regions of the solar atmosphere. Recently, Chmielewski et al. [22] modelled numerically the impulsively excited non-linear Alfvén waves in solar coronal holes, and concluded that such waves may result in the observed spectral line broadening in the solar atmosphere [17] have also reported that the resonant energy conversion from Alfvén (transverse) 
to acoustic (longitudinal) waves in the lower solar atmosphere where plasma $\beta$ becomes unity. This effect can be responsible for the spectral line width variation and narrowing due to the dissipation of Alfvén waves. However, this theory could only demonstrate the most likely physical scenario behind the line-width reduction as observed only by O'Shea et al. [19] in polar coronal hole beyond 1.21 solar radii. Additionally, there is not enough observational evidences that provide the resonant energy conversion in the solar corona between Alfvén as well as magnetoacoustic waves [24-25].

The above described recent discoveries of Alfvén waves in the solar atmosphere well-justify extensive studies of the waves that were performed by numerous investigators in the last few decades both analytically and numerically [26-28], and references therein. These studies covered both linear [29] and non-linear [30-32] Alfvén waves, and different aspects of the wave generation, propagation and dissipation were investigated. The specific objectives of these studies were to understand the role of Alfvén waves in the atmospheric heating and localized plasma dynamics. In this paper, we numerically investigate the behavior of small-amplitude (linear) and large-amplitude (nonlinear) Alfvén waves in a model of the solar atmosphere with the weakly curved magnetic field that mimics open magnetic field structure of the solar corona. Such magnetic field configurations, which are commonly known as coronal funnels, can be widely applicable to the polar coronal holes, expanding open arches near the boundary of the coronal holes in the quiet-Sun as well as fanloop arches, where plasma and plasma flows are observed. In Sect. 2 we discuss the numerical simulation of pulse-driven Alfvén waves. We present the results of numerical simulations in Sect. 3. Our discussion of the obtained results and conclusions are given in the last section of this paper.

\section{Numerical simulation of the pulse-driven Alfvén waves in coronal funnels}

Our model of the solar atmosphere consists of a gravitationally-stratified plasma in a weakly curved magnetic field configuration that mimics the coronal funnels in the solar atmosphere. The model is described by the following set of ideal magnetohydrodynamic (MHD) equations:

$$
\begin{aligned}
& \frac{\partial \varrho}{\partial t}+\nabla(\varrho \boldsymbol{V})=0, \\
& \varrho \frac{\partial \boldsymbol{V}}{\partial t}+\varrho(\boldsymbol{V} \cdot \nabla) \boldsymbol{V}=-\nabla p+\frac{1}{\mu}(\nabla \times \boldsymbol{B}) \times \boldsymbol{B}+\varrho \boldsymbol{g},
\end{aligned}
$$$$
\frac{\partial \boldsymbol{B}}{\partial t}=\nabla \times(\boldsymbol{V} \times \boldsymbol{B}),
$$$$
\nabla \cdot \boldsymbol{B}=0
$$$$
\frac{\partial p}{\partial t}+\boldsymbol{V} \cdot \nabla p=-\gamma p \nabla \cdot \boldsymbol{V}
$$$$
p=\frac{k_{\mathrm{B}}}{m} \varrho T \text {. }
$$

Here $\varrho$ is mass density, $\boldsymbol{V}$ and $\boldsymbol{B}$ are vectors of the flow velocity and the magnetic field respectively, $p$ is gas pressure, $\gamma=5 / 3$ is the adiabatic index, $\mu$ is the magnetic permeability of the plasma, $\boldsymbol{g}=(0,-g, 0)$ is a vector of gravitational acceleration with its value $g=274 \mathrm{~m} \mathrm{~s}^{-2}$, $T$ is a temperature, $m$ is a mean particle mass and $k_{\mathrm{B}}$ is a Boltzmann constant.

We consider a $2.5 \mathrm{D}$ model of the solar atmosphere with an invariant coordinate $(\partial / \partial z=0)$ and varying the $z$-components of velocity $\left(V_{z}\right)$ and magnetic field $\left(B_{z}\right)$ with $x$ and $y$. The solar atmosphere is in static equilibrium $\left(\boldsymbol{V}_{\mathrm{e}}=\mathbf{0}\right)$ with force- and current-free magnetic field, i.e.,

$$
\left(\nabla \times \boldsymbol{B}_{\mathrm{e}}\right) \times \boldsymbol{B}_{\mathrm{e}}=\mathbf{0}, \quad \nabla \times \boldsymbol{B}_{\mathrm{e}}=\mathbf{0} .
$$

Equilibrium quantities are described by the subscript $\mathrm{e}$.

In our model of atmosphere a curved magnetic field of the coronal funnel

$$
\boldsymbol{B}_{\mathrm{e}}=\nabla \times \boldsymbol{A}_{\mathrm{e}},
$$

is given by the following magnetic flux function:

$$
\boldsymbol{A}_{\mathrm{e}}=\Lambda_{\mathrm{B}} B_{0} \cos \left(\frac{x}{\Lambda_{\mathrm{B}}}\right) \exp \left(-\frac{y-y_{\mathrm{r}}}{\Lambda_{\mathrm{B}}}\right) \hat{\boldsymbol{z}},
$$

where $\hat{\boldsymbol{z}}$ is a unit vector along the $z$-direction and $B_{0}$ is the magnetic field at the reference level, $y=y_{\mathrm{r}}$, which is chosen at $y_{\mathrm{r}}=10 \mathrm{Mm}$. We set and hold fixed $B_{0}$ in such a way that the Alfvén speed, $c_{\mathrm{A}}=$ $B_{0} / \sqrt{\mu \varrho_{\mathrm{e}}\left(y=y_{\mathrm{r}}\right)}$ is ten times higher than the sound speed, $c_{\mathrm{s}}=\sqrt{\gamma p_{\mathrm{e}}\left(y=y_{\mathrm{r}}\right) / \varrho_{\mathrm{e}}\left(y=y_{\mathrm{r}}\right)}$. Such a choice of $\boldsymbol{B}_{\text {e }}$ results in Eq. (7) being satisfied. Here $\Lambda_{\mathrm{B}}=2 L / \pi$ denotes the magnetic scale-height and $L$ is a half of the magnetic arcade width. As we aim to model a weakly expanding coronal funnels, we take $L=75 \mathrm{Mm}$ and keep it fixed in our calculations. For this setting, the magnetic field lines are weakly curved and represent the open and expanding field lines similar to the coronal holes.

As a result of Eq. (7), the pressure gradient is balanced by the gravity force

$$
-\nabla p_{\mathrm{e}}+\varrho_{\mathrm{e}} \boldsymbol{g}=\mathbf{0} \text {. }
$$

Using the ideal gas law of Eq. (6) and the $y$-component of the hydrostatic pressure balance indicated by Eq. (10), we express equilibrium gas pressure and mass density as

$$
p_{\mathrm{e}}(y)=p_{0} \exp \left(-\int_{y_{\mathrm{r}}}^{y} \frac{\mathrm{d} y^{\prime}}{\Lambda\left(y^{\prime}\right)}\right), \quad \varrho_{\mathrm{e}}(y)=\frac{p_{\mathrm{e}}(y)}{g \Lambda(y)},
$$

where

$$
\Lambda(y)=\frac{k_{\mathrm{B}} T_{\mathrm{e}}(y)}{m g}
$$

is the pressure scale-height, and $p_{0}$ denotes the gas pressure at the reference level.

We consider a realistic model of the plasma temperature profile [33] displayed in Fig. 1 (left part). Temperature attains a value of about $6 \times 10^{3} \mathrm{~K}$ at $y=1.5 \mathrm{Mm}$ and it increases up to about $1.5 \times 10^{6} \mathrm{~K}$ in the solar corona at $y=10 \mathrm{Mm}$. Higher up the temperature is assumed to be constant. The temperature profile determines uniquely the equilibrium mass density and gas pressure profiles. Both $\varrho_{\mathrm{e}}(y)$ and $p_{\mathrm{e}}(y)$ experience a sudden drop at the transition region that is located at $y \approx 2.7 \mathrm{Mm}$. 
In this model the Alfvén speed, $c_{\mathrm{A}}$, varies only with $y$ and is expressed as follows:

$$
c_{\mathrm{A}}(y)=\frac{B_{0} \mathrm{e}^{-\frac{y-y_{\mathrm{r}}}{\Lambda_{\mathrm{B}}}}}{\sqrt{\mu \varrho_{\mathrm{e}}(y)}} .
$$

Its profile is displayed in Fig. 1, right part. Let us note that the Alfvén speed in the chromosphere, $c_{\mathrm{A}}(y=$ $1.75 \mathrm{Mm})$, is about $25 \mathrm{~km} \mathrm{~s}^{-1}$. The Alfvén speed rises abruptly through the transition region reaching a value of $c_{\mathrm{A}}(y=10 \mathrm{Mm})=10^{3} \mathrm{~km} \mathrm{~s}^{-1}$ (Fig. 1, right part). The growth of $c_{\mathrm{A}}(y)$ with height results from a faster decrement of $\varrho_{\mathrm{e}}(y)$ than $B_{\mathrm{e}}(y)$ with the height. We see that this profile is more realistic than the one used by Murawski and Musielak [29].
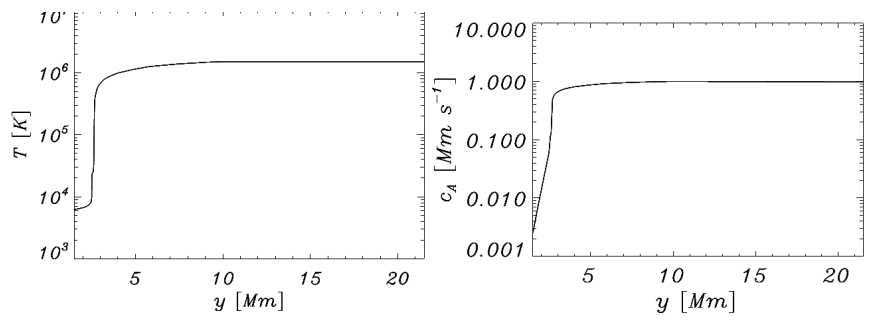

Fig. 1. Equilibrium profile of the temperature (left part) and the Alfvén speed (right part).

The realistic solar atmosphere above the polar coronal holes, the fan-like arches near the boundary of active regions, quiet-Sun expanding flux-tubes, reveal complexity of its plasma and magnetic field structure. The magnetic field configuration in such weakly expanding magnetized structures can be approximated by expanding coronal funnels in the lower part of the atmosphere and comparatively smooth open field lines in its upper part $[34,35]$. This implies that the field structure and magnetic scale-height vary in such coronal funnels. Moreover, the field configuration changes from dipolar to multipolar during the transition from the solar minimum to its maximum. Despite these well-known variations, we assume that the magnetic field scale height is fixed at a reasonable value of $75 \mathrm{Mm}$ in our simulation domain, which represents the weakly curved and open field lines of the coronal funnels in the solar atmosphere. We want to point out that this assumption does not affect the validity of our numerical results as these weakly expanding coronal funnels can extend up to few hundreds megameters, while they can be few megameter wide in the horizontal direction $[34,35]$.

Equations (1)-(6) are solved numerically with a use of the FLASH code [36]. This code implements a second-order unsplit Godunov solver with various slope limiters and Riemann solvers as well as adaptive mesh refinement (AMR). We use the minmod slope limiter and the Roe Riemann solver [37]. The simulation box is set as $(-5 \mathrm{Mm}, 5 \mathrm{Mm}) \times(1 \mathrm{Mm}, 81 \mathrm{Mm})$ and there are imposed fixed in time boundary conditions for all plasma quantities in $x$ - and $y$-directions, while all plasma quantities remain invariant along the $z$-direction. However,
$V_{z}$ and $B_{z}$, in general, are different than zero. In our present work, we use AMR grid with a minimum (maximum) level of refinement set to $3(8)$. The refinement strategy is based on controlling the numerical errors in mass density.

Now, in order to get a fine grid, our simulation region is enshroud by eight equilateral blocks that corresponds to zeroth level of grid refinement. Then, some these blocks are divided into $2^{n}$ smaller blocks, where $n=2$ denotes space dimension of our numerical model. In this way, we raise the level of refinement and we get an initial, non-uniform numerical grid. Our simulation box is covered by the numerical grid, which is finer below the transition region and along $x=0$, the path of Alfvén wave propagation, and rare, but dense enough in the solar corona. This results in an excellent resolution of steep spatial profiles and greatly reduces the numerical diffusion at these locations. As every numerical block consists of $8 \times 8$ identical numerical cells, we reach the finest spatial resolution of $39 \mathrm{~km}$. Let us note that our simulation region is chosen large and enough to observe wide pulse Alfvén waves propagating along magnetic field lines. Therefore, we aim to model a general coronal funnel structures where such waves are evolved.

We perturb initially (at $t=0 \mathrm{~s}$ ) the equilibrium model by a Gaussian pulse in the $z$-component of velocity given by

$$
V_{z}(x, y, t=0)=A_{v} \exp \left(-\frac{\left(x-x_{0}\right)^{2}+\left(y-y_{0}\right)^{2}}{w^{2}}\right),
$$

where $A_{v}$ is the amplitude of the pulse, $\left(x_{0}, y_{0}\right)$ is its initial position and $w$ denotes its width. We set $w=0.2 \mathrm{Mm}$ $\left(x_{0}=0, y_{0}=1.75\right) \mathrm{Mm}$ and consider three cases: (a) $A_{v}=5 \mathrm{~km} \mathrm{~s}^{-1}$; (b) $A_{v}=10 \mathrm{~km} \mathrm{~s}^{-1}$; (c) $A_{v}=40 \mathrm{~km} \mathrm{~s}^{-1}$. Note that the initial magnetic field is initially not perturbed directly by the initial pulse. However, magnetic field will be altered in a while as the energy will be transferred linearly from $V_{z}$ into $B_{z}$. As a result of that, the initial pulse we implemented mimics the real case. Note that in the $2.5 \mathrm{D}$ model we developed, the Alfvén waves decouple from magnetoacoustic waves and it can be described by $V_{z}(x, y, t)$. As a result, the initial pulse triggers Alfvén waves that in the linear limit are described by the wave equation

$$
\frac{\partial^{2} V_{z}}{\partial t^{2}}=c_{\mathrm{A}}^{2}(y) \frac{\partial^{2} V_{z}}{\partial y^{2}} \text {. }
$$

\section{Results of the numerical simulation of Alfvén waves in coronal funnels}

We simulate both linear $\left(A_{v}=5 \mathrm{~km} \mathrm{~s}^{-1}\right)$ and non-linear $\left(A_{v}=10 \mathrm{~km} \mathrm{~s}^{-1}\right.$ and $\left.A_{v}=40 \mathrm{~km} \mathrm{~s}^{-1}\right)$ impulsively excited Alfvén waves and investigate their propagation along the open and expanding magnetic field lines of coronal funnels. We categorize linear (non-linear) waves as those whose amplitude is significantly smaller, by $10 \%$, than (or comparable to) a local Alfvén velocity. The amplitude with few kilometers per second will 
generate the linear Alfvénic perturbations, while the amplitude with few tens of kilometer per second is responsible for the non-linear Alfvén waves. The results are compared to those previously obtained by Chmielewski et al. [22]. It should be noted that the effect of inhomogeneities across magnetic field lines is not included in our approach. The waves are generated by the transversal velocity pulse perpendicular to the magnetic isosurface $(\mathrm{X}-\mathrm{Y})$ in the $z$-direction and we compute the maximum transversal velocity $\left(V_{z}\right)$ at different heights in the simulation domain.

We study the case of an initial localized pulse that is launched in $V_{z}(x, y)$ at $t=0 \mathrm{~s}$ perpendicular to the isosurface of the approximately open (weakly curved) and expanding magnetic field configuration. This pulse is described by Eq. (14). Examples of spatial profiles of $V_{z}$ and the corresponding time-signatures obtained in our numerical simulation of Alfvén waves are shown in Figs. 2 and 3 , respectively.

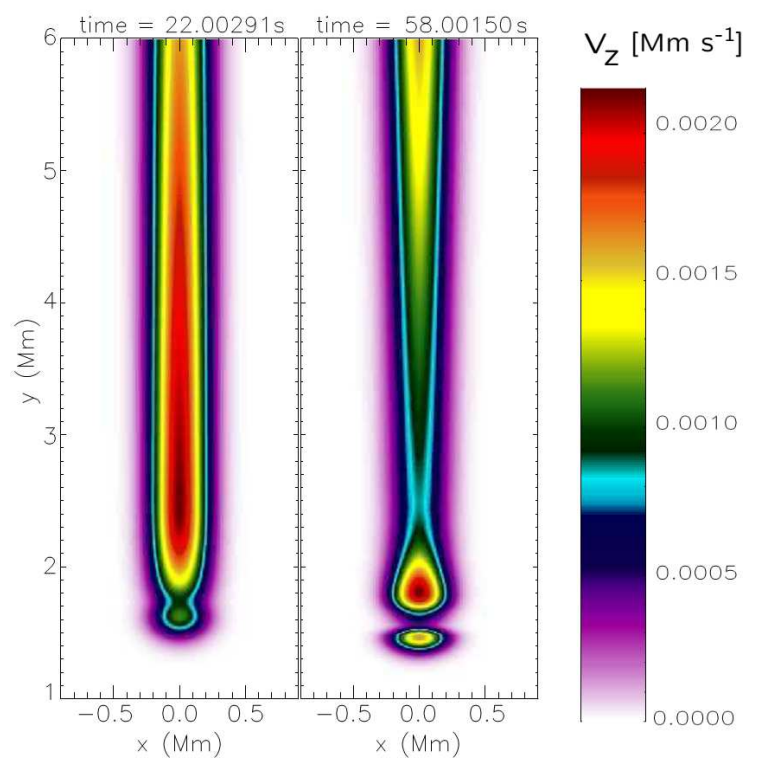

Fig. 2. Transverse velocity $V_{z}$ profiles at $t=22 \mathrm{~s}$ and $t=58 \mathrm{~s}$ for $A_{v}=5 \mathrm{~km} \mathrm{~s}^{-1}$.

First, we consider the pulse amplitude $A_{v}=5 \mathrm{~km} \mathrm{~s}^{-1}$ for the simulation of the pulse-driven linear Alfvén waves in the coronal funnel. Perturbations in $V_{z}$ propagate essentially along magnetic field lines, which is displayed on spatial profiles of $V_{z}$ at $t=22 \mathrm{~s}$ and $58 \mathrm{~s}$ (Fig. 2). A part of the whole simulation region is illustrated. The initial pulse launched at $y_{0}=1.75 \mathrm{Mm}$ decouples into two counter-propagating waves. The amplitude of downwardly propagating waves falls off in time and these waves subside. On the other hand, upwardly propagating Alfvén waves experience an acceleration at the transition region as $c_{\mathrm{A}}(y)$ increases up to $1 \mathrm{Mm} \mathrm{s}^{-1}$ there (Fig. 1, right) and their profile becomes elongated along the vertical direction. Above the transition region, Alfvén waves propagate with essentially constant amplitude and veloc-

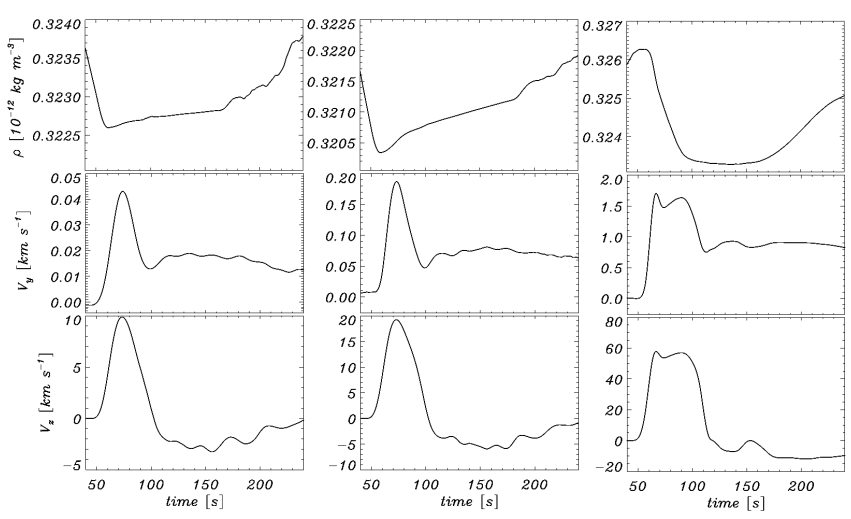

Fig. 3. Results of the numerical simulation of pulse-driven Alfvén waves: time-signatures of $\rho, V_{y}$ and $V_{z}$ for $A_{v}=5 \mathrm{~km} \mathrm{~s}^{-1}$ (left column), $A_{v}=10 \mathrm{~km} \mathrm{~s}^{-1}$ (middle column) and $A_{v}=40 \mathrm{~km} \mathrm{~s}^{-1}$ (right column) collected at $(x=0, y=51) \mathrm{Mm}$.

ity. A part of the wave signal reflected at the transition region is well seen at $y \approx 2 \mathrm{Mm}$ at $t=58 \mathrm{~s}$ (Fig. 2, right part).

Parts in the left column of Fig. 3 illustrate typical features of the linear Alfvén wave. Here, the profiles of the mass density and $y$-component of velocity exhibit only small variations in time. The growth of $V_{y}$ is by two orders of magnitude smaller than transverse velocity and it is directly associated with the Alfvén waves, which reach the detection point $(x=0 \mathrm{Mm}, y=51 \mathrm{Mm})$ at $t \approx 55 \mathrm{~s}$. The mass density profiles exhibit a negligible decline during the Alfvén wave passing. It is significant that the Alfvén waves while passing through the solar atmosphere experience a compact shape (Fig. 3, bottom parts $)$. The time-signature of $V_{z}(x=0 \mathrm{Mm}, y=51 \mathrm{Mm})$ for $A_{v}=5 \mathrm{~km} \mathrm{~s}^{-1}$, illustrated in Fig. 3 (bottom-left part), were found to be similar to that obtained by $\mathrm{Mu}-$ rawski and Musielak [29] (cf. their Fig. 6).

In the middle column of Fig. 3, the time-signatures of the Alfvén waves, which are launched by the initial pulse with $A_{v}=10 \mathrm{~km} \mathrm{~s}^{-1}$ are presented. Let us note that the time-signature of the Alfvén waves for $A_{v}=10 \mathrm{~km} \mathrm{~s}^{-1}$ has a similar form as in the linear case. However, the wave intensifies flow along $y$-direction (Fig. 3, middle-center part) twice in comparison to pulse with $A_{v}=$ $5 \mathrm{~km} \mathrm{~s}^{-1}$.

We also simulate the Alfvén wave using the large transversal pulse $A_{v}=40 \mathrm{~km} \mathrm{~s}^{-1}$ that generates the large-amplitude, non-linear Alfvén waves [22] to make comparison with the Alfvén waves driven by two other pulses. The time-signatures of $V_{z}$ for $A_{v}=40 \mathrm{~km} \mathrm{~s}^{-1}$ collected at $(x=0, y=51) \mathrm{Mm}$ are shown in the right column of Fig. 3. This wave experiences non-linear effects, as a result of which vertical flow (Fig. 3, middle-right part) is driven by a ponderomotive force. The time-signature of $V_{z}$ exhibits a double peak shape. This deformation arises as a result of partial reflection of the 
Alfvén waves from the transition region. The reflected signal reaches a dense photospheric plasma and reflects back towards the solar corona. For a larger initial pulse amplitude this effect is more important. Let us note that even for the Alfvén waves triggered by the pulse amplitude $A_{v}=10 \mathrm{~km} \mathrm{~s}^{-1}$ the Gaussian shape of the wave signal is deformed on its right side (Fig. 3, bottom-middle part).

In general, time-signatures of large-amplitude Alfvén waves exhibit similar behaviour as of the linear Alfvén wave with $A_{v}=5 \mathrm{~km} \mathrm{~s}^{-1}$. We observe effects of the non-linear Alfvén wave decoupling from magnetoacoustic waves in $V_{y}$ whose magnitude grows with $A_{v}$. The spatial profile of $V_{y}$ for $A_{v}=40 \mathrm{~km} \mathrm{~s}^{-1}$ (Fig. 3, middle-right part) is like for $A_{v}=5 \mathrm{~km} \mathrm{~s}^{-1}$ or $A_{v}=10 \mathrm{~km} \mathrm{~s}^{-1}$, although in the case (c) it is forty times bigger than in the linear case (a) and suffer deformation coming from a partial reflection of the Alfvén wave at the transition region.

Let us note that the mass density variations for the Alfvén waves triggered by the initial pulses with $A_{v}=$ $5 \mathrm{~km} \mathrm{~s}^{-1}, A_{v}=10 \mathrm{~km} \mathrm{~s}^{-1}$ and $A_{v}=40 \mathrm{~km} \mathrm{~s}^{-1}$ (cf. Fig. 3 , top parts) are insignificantly small, in particular for case (a) and (b), which are twice smaller in comparison to the case (c).

\section{Discussion and conclusions}

In our pulse driven Alfvén wave model, the pulse of a small and large amplitudes are launched above the solar photosphere in the weakly expanding coronal funnels. The pulse splits into the upward and backward propagating wavetrains in the overlying solar atmosphere. The upward moving pulse train results in the instantaneous displacement of the field lines in perpendicular plane away and towards the line-of-sight. This effect generates the transverse velocity. The pulse driven linear $\left(A_{v}=5 \mathrm{~km} \mathrm{~s}^{-1}\right)$ and non-linear $\left(A_{v}=10 \mathrm{~km} \mathrm{~s}^{-1}\right.$ and $A_{v}=40 \mathrm{~km} \mathrm{~s}^{-1}$ ) Alfvén waves exhibit different contributions in the transverse velocity $V_{z}$ component at a particular height, and therefore they can play different roles in the local energy budget of the solar atmosphere. A typical granular motions can freely trigger the linear Alfvén wave with pulse amplitude $A_{v}=5 \mathrm{~km} \mathrm{~s}^{-1}$. Whereas a generation of non-linear Alfvén wave is possible in processes of microscopic magnetic reconnection like micro- or nano-flares, where released kinetic energy disturbs plasma in velocity generating MHD waves [38].

In theory, the maximum energy flux density carried out by such Alfvén waves under the Wentzel-KramersBrillouin (WKB) approximation is [39]:

$$
F \approx \rho \times V_{z}^{2} \times c_{\mathrm{A}}
$$

where $\rho$ is the density at the particular height, while the $c_{\mathrm{A}}$ is the local phase speed of the Alfvén waves. It should be noted that we do not calculate the energy flux distribution per degree of freedom, as we generalize the magnetic field configuration where such modes are excited.
For the linear Alfvén waves $\left(A_{v}=5 \mathrm{~km} \mathrm{~s}^{-1}\right)$ in the coronal funnel, and by considering $\rho_{\mathrm{e}}=0.323 \times 10^{-15} \mathrm{~g} \mathrm{~cm}^{-3}$, $V_{z}=10 \mathrm{~km} \mathrm{~s}^{-1}$ (cf. Fig. 3, left column) as well as $c_{\mathrm{A}}=10^{3} \mathrm{~km} \mathrm{~s}^{-1}$ at $y=51 \mathrm{Mm}(1.07 \mathrm{Ro})$ in the inner part of the coronal funnel, we can estimate the maximum energy flux carried out by such waves as $F_{\max } \approx$ $3.0 \times 10^{4} \mathrm{erg} \mathrm{cm}^{-2} \mathrm{~s}^{-1}$.

If the coronal funnel of the similar physical and magnetic field configuration exists in the polar coronal holes, then the energy carried out by such impulsively excited Alfvén waves will almost be sufficient to fulfill the energy requirement of the inner corona [39]. If the coronal funnel will be existing in the quiet-Sun or in form of active region loop arches, then such waves may only partially fulfill the energy requirements. For the non-linear Alfvén waves $\left(A_{v}=10 \mathrm{~km} \mathrm{~s}^{-1}\right)$ in the coronal funnels, and by considering $\rho_{\mathrm{e}}=0.321 \times 10^{-15} \mathrm{~g} \mathrm{~cm}^{-3}, V_{z}=20 \mathrm{~km} \mathrm{~s}^{-1}$ (cf. the middle column in Fig. 3) as well as $c_{\mathrm{A}}=10^{3} \mathrm{~km} \mathrm{~s}^{-1}$ at $y=51 \mathrm{Mm}$ (1.07 Ro) in its inner part, we can estimate the maximum energy flux carried out by such waves as $F_{\max } \approx 1.3 \times 10^{5} \mathrm{erg} \mathrm{cm}^{-2} \mathrm{~s}^{-1}$. This wave energy flux will be sufficient to fulfill the energy requirement of the inner corona [40]. However, for the coronal funnel that exists in form of active region loop arches, the computed wave energy flux only partially fulfills the required energy budget of the corona.

Now, for the non-linear Alfvén waves $\left(A_{v}=40 \mathrm{~km} \mathrm{~s}^{-1}\right)$ in the coronal funnels, and by considering $\rho_{\mathrm{e}}=0.325 \times$ $10^{-15} \mathrm{~g} \mathrm{~cm}^{-3}, V_{z}=60 \mathrm{~km} \mathrm{~s}^{-1}$ (cf. Fig. 3, right column) as well as $c_{\mathrm{A}}=10^{3} \mathrm{~km} \mathrm{~s}^{-1}$ at a height of $51 \mathrm{Mm}$ (1.07 Ro) in its inner part, we can estimate the maximum energy flux carried out by such waves as $F_{\max } \approx$ $1.2 \times 10^{6} \mathrm{erg} \mathrm{cm}^{-2} \mathrm{~s}^{-1}$, which can again be sufficient to fulfill the energy requirement of the inner corona [40]. Chmielewski et al. [22] studied in detail the role of such non-linear Alfvén waves in the polar coronal holes and their role in the observed spectral line broadening.

It should be noted that our calculation of the WKB part of the energy are performed only to make comparison with the wave energy computed for the linear waves. However, applications of the WKB method are limited because of abrupt variations of the plasma parameters in the stratified solar atmosphere whose spatial scales can be at a comparable magnitude to a typical wavelength. Nevertheless, we apply the WKB method in the linear regime, i.e. we ignore the higher order terms, which are related to higher-order mass density variations. As a result, we even underestimate the energy flux evaluations and the non-linear high-amplitude Alfvén waves are sufficient to fulfill the localized coronal energy losses.

In conclusion, we showed that the linear pulse-driven Alfvén waves can sufficiently power the inner corona above the polar coronal holes (for $A_{v}=5 \mathrm{~km} \mathrm{~s}^{-1}$ ), while the non-linear waves (for $A_{v}=10 \mathrm{~km} \mathrm{~s}^{-1}$ carry enough energy to fulfill the energy requirement in solar coronal holes as well as quiet-Sun energy losses. Our results clearly demonstrated that non-linear Alfvén waves $\left(A_{v}=40 \mathrm{~km} \mathrm{~s}^{-1}\right)$ can power the inner corona in the ex- 
panding coronal funnels existing anywhere, in the coronal holes, quiet-Sun, as well as in form of active region fan-like loop arches. In general, the Alfvén wave dissipation occurs either in the distant part of the corona [41], or by some unique processes (e.g., phase-mixing, resonant absorption) in the solar atmosphere [42, 43]. Therefore, the lower part of the solar atmosphere above the polar corona may be the ideal place for the undamped growth of linear and non-linear Alfvén waves, and sufficiently large amplitude Alfvén waves may supply (and damp) their energy in the outer part of the corona and can heat the solar wind ions [44].

In the corona, the linear (e.g., $A_{v}=5 \mathrm{~km} \mathrm{~s}^{-1}$ ) and non-linear Alfvén waves (e.g., $A_{v}=40 \mathrm{~km} \mathrm{~s}^{-1}$ ) can carry sufficient amount of energy, and provide momentum to accelerate the solar wind plasma along the expanding magnetic field lines of the coronal funnels (e.g., coronal hole, and quiet-sun flux-tubes, respectively) [13]. Despite of polar coronal holes and quiet-Sun open magnetic field structures, the solar wind outflows and large-scale flows are also observed in the open magnetic arches near the boundary of the active regions [45]. Therefore, the large-amplitude, non-linear, pulse-driven Alfvén waves can provide sufficient energy to such kind of flows above the active regions in the expanding coronal funnels.

\section{Acknowledgments}

This work has been supported by NSF under the grant AGS 1246074 (K.M. and Z.E.M.) and by the Alexander von Humboldt Foundation (Z.E.M.). The software used in this work was in part developed by the DOE-supported ASCI/Alliance Center for Astrophysical Thermonuclear Flashes at the University of Chicago. A.K.S. thanks Shobhna Srivastava for patient encouragement. A.K.S. acknowledges the financial support from DST-RFBR-P117 project. AKS thanks Prof. K. Murawski to provide the financial support for his visit to UMCS, Lublin in September 2013 where present and various others collaborative works have been executed.

\section{References}

[1] B.N. Dwivedi, A.K. Srivastava, Curr. Sci. 98, 295 (2010).

[2] H. Alfvén, Nature 405, 150 (1942).

[3] T.J. Okamoto, S. Tsuneta, T.E. Berger, K. Ichimoto, Y. Katsukawa, B.W. Lites, S. Nagata, K. Shibata, T. Shimizu, R.A. Shine, Y. Suematsu, T.D. Tarbell, A.M. Title, Science 318, 1577 (2007).

[4] B. De Pontieu, S.W. McIntosh, M. Carlsson, V.H. Hansteen, T.D. Tarbell, C. Schrijver, A.M. Title, R.A. Shine, S. Tsuneta, Y. Katsukawa, K. Ichimoto, Y. Suematsu, T. Shimizu, S. Nagata, Science $\mathbf{3 1 8 ,}$ 1574 (2007)

[5] J.W. Cirtain, L. Golub, L. Lundquist, A. van Ballegooijen, A. Savcheva, M. Shimojo, E. DeLuca, S. Tsuneta, T. Sakao, K. Reeves, M. Weber, R. Kano, N. Narukage, K. Shibasaki, Science 318, 1580 (2007).
[6] R. Erdélyi, V. Fedun, Science 318, 1572 (2007).

[7] T. Van Doorsselaere, V.N. Nakariakov, E. Verwichte, Astrophys. J. Lett. 676, L73 (2008).

[8] M. Goossens, J. Terradas, J. Andries, I. Arregui, J.L. Ballester, Astron. Astrophys. 503, 213 (2009).

[9] M. Goossens, J. Andries, R. Soler, T. Van Doorsselaere, I. Arregui, J. Terradas, Astrophys. J. 753, 111 (2012).

[10] H. Tian, S.W. McIntosh, T. Wang, L. Ofman, B. De Pontieu, D.E. Innes, P. Hardi, Astrophys. J. 759, 144 (2012).

[11] D.B. Jess, M. Mathioudakis, R. Erdlyi, P.J. Crockett, F.P Keenan, D.J. Christian, Science 323, 1582 (2009).

[12] S. Tomczyk, S.W. McIntosh, S.L. Keil, P.G. Judge, T. Schad, D.H. Seeley, J. Edmondson, Science 317 , 1192 (2007).

[13] C.-Y. Tu, C. Zhou, E. Marsch, L.-D. Xia, L. Zhao, J.-X. Wang, K. Wilhelm, Science 308, 519 (2005).

[14] B.N. Dwivedi, A.K. Srivastava, Sol. Phys. 237, 143 (2006).

[15] A.K. Srivastava, B.N. Dwivedi, J. Astrophys. Astron. 28, 1 (2007).

[16] D.M. Hassler, G.J. Rottman, E.C. Shoub, T.E. Holzer, Astrophys. J. Lett. 348, L77 (1990).

[17] D. Banerjee, L. Teriaca, J.G. Doyle, K. Wilhelm, Astron. Astrophys. 339, 208 (1998).

[18] T.G. Moran, Astrophys. J. 598, 657 (2003).

[19] E. O'Shea, D. Banerjee, J.G. Doyle, Astron. Astrophys. 436, L43 (2005).

[20] L. Dolla, J. Solomon, Astron. Astrophys. 483, 271 (2008).

[21] A. Bemporad, L. Abbo, Astrophys. J. 110, 751 (2012).

[22] P. Chmielewski, A.K. Srivastava, K. Murawski, Z.E. Musielak, Monthly Notic. Roy. Astron. Soc. 428, 40 (2013).

[23] T.V. Zaqarashvili, R. Oliver, J.L. Ballester, Astron. Astrophys. 456, L13 (2006).

[24] A.K. Srivastava, B.N. Dwivedi, Monthly Notic. Roy. Astron. Soc. 405, 2317 (2010).

[25] R.T.J. McAteer, P.T. Gallagher, D.R. Williams, M. Mathioudakis, D.S. Bloomfield, K.J.H. Phillips, F.P. Keenan, Astrophys. J. 587, 806 (2003).

[26] Z.E. Musielak, R.L. Moore, Astrophys. J. 452, 434 (1995).

[27] B. Roberts, SOHO 13 Waves, Oscillations and SmallScale Transients Events in the Solar Atmosphere: Joint View from SOHO and TRACE 547, 1 (2004).

[28] P. Antolin, K. Shibata, T. Kudoh, D. Shiota, D. Brooks, The Second Hinode Science Meeting: Beyond Discovery-Toward Understanding 415, 247 (2009).

[29] K. Murawski, Z.E. Musielak, Astron. Astrophys. 518, A37 (2010).

[30] A. Verdini, M. Velli, Astrophys. J. 662, 669 (2007).

[31] A. Verdini, M. Velli, E. Buchlin, Astrophys. J. Lett. 700, L39 (2009). 
[32] T. Matsumoto, K. Shibata, Astrophys. J. 710, 1857 (2010).

[33] J.E. Vernazza, E.H. Avrett, R. Loeser, Astrophys. J. Suppl. Series 45, 635 (1981).

[34] M. Banaszkiewicz, W.I. Axford, J.F. McKenzie, Astron. Astrophys. 337, 940 (1998).

[35] P. Hackenberg, E. Marsch, G. Mann, Astron. Astrophys. 360, 1139 (2000)

[36] B. Fryxell, K. Olson, P. Ricker, F.X. Timmes, M. Zingale, D.Q. Lamb, P. MacNeice, R. Rosner, J.W. Truran, H. Tufo, Astrophys. J. Suppl. Series 131, 273 (2000).

[37] E.F. Toro, Int. J. Numer. Meth. Fluids 52, 433 (2006).

[38] H.S. Hudson, Sol. Phys. 133, 357 (1991).
[39] T. Kudoh, K. Shibata, Astrophys. J. 514, 493 (1999).

[40] G.L. Withbroe, R.W. Noyes, Ann. Rev. Astron. Astrophys. 15, 363 (1977).

[41] E.N. Parker, Astrophys. J. 372, 719 (1991).

[42] J. Heyvaerts, E.R. Priest, Astron. Astrophys. 117, 220 (1983).

[43] R. Erdelyi, M. Goossens, Astron. Astrophys. 294, 575 (1995).

[44] L. Ofman, J.M. Davila, Astrophys. J. 553, 935 (2001).

[45] L.K. Harra, T. Sakao, C.H. Mandrini, H. Hara, S. Imada, P.R. Young, L. van Driel-Gesztelyi, D. Baker, Astrophys. J. 676, L147 (2008). 